\title{
Perks of percutaneous cryoablation
}

Two new studies support an expansion of the role of percutaneous cryoablation in the management of renal masses.

The use of ablative techniques was originally restricted to those patients harboring small kidney tumors for whom the surgical standard of open partial nephrectomy was contraindicated. Now, patients without such contraindications frequently request that they be considered for radiofrequency ablation or cryoablation. The lessinvasive nature of these procedures, and relatively short recovery time, are key drivers of this demand.

The experience of Jaime Landman and colleagues supports the perception that percutaneous cryoablation is a safe and effective means of managing renal tumors up to $3 \mathrm{~cm}$ in diameter. In their series of 99 posteriorly located masses (50\% malignant) in 90 patients, the treatment failure and major complication rates were $9 \%$ and $2 \%$, respectively. Failures were successfully remedied by a second round of percutaneous cryoablation in all but one patient. No patient had died from disease during a median follow-up period of 11 months.

Landman et al. also reviewed their data on laparoscopic cryoablation, of both anterior and posterior lesions ( $n=97 ; 60 \%$ malignant). Again, the incidences of major complications and treatment failure were low ( $4 \%$ and $3 \%$, respectively). Cancer-specific survival after a median of 12 months was $100 \%$. Mean freeze time was significantly less during laparoscopic cryoablation, but patients who were treated using this approach remained in hospital for longer ( 1.5 days compared with 0.1 days for percutaneous cryoablation).

So, the outcomes of percutaneous cryoablation of small renal tumors are favorable, at least in the short term. Grant Schmit and colleagues contend that this conclusion also applies to much larger lesions. "Percutaneous renal cryoablation in patients with renal masses larger than $3 \mathrm{~cm}$ can be done effectively and with far fewer complications than might be expected", he reports.

Schmit et al. base this assertion on their experience of 108 patients with 110 tumors ranging in size from $3 \mathrm{~cm}$ to $8 \mathrm{~cm}$. Nine major adverse events occurred. There were three technical failures, all as a result of incomplete ablation of the deepest portion of a central tumor. Local recurrence was not detected in any patient during an average of 15 months of postoperative monitoring.

The authors emphasize the importance of operator experience to the success of percutaneous cryoablation of large kidney masses. The six interventional radiologists that performed the procedures had been applying the technique for between 3 and 10 years. Schmit et al. also believe that general anesthesia, as opposed to moderate sedation, of patients is associated with superior outcomes. Further, the team has collected preliminary data which show that pretreatment with selective arterial tumor embolization can minimize bleeding during ablation of lesions larger than $5 \mathrm{~cm}$.

Percutaneous cryoablation is a relatively new modality. Its role in the management of renal masses remains controversial. Proving that the favorable short-term results reported here persist over time in a large cohort will be essential to safe and efficacious usage.

Suzanne J. Farley

Original articles Mues, A. C. et al. Comparison of percutaneous and laparoscopic renal cryoablation for small $(<3.0 \mathrm{~cm})$ renal masses. J. Endourol. doi:10.1089/end.2010.0067 | Schmit, G. D. et al. Percutaneous cryoablation of renal masses $\geq 3 \mathrm{~cm}$ : efficacy and safety in treatment of 108 patients. J. Endourol. doi:10.1089/end.2009.0328 